\title{
Negativemissionstechnologien und ihre Verortung im Regelsystem internationaler Klimapolitik*
}

\author{
Till Markus/Romina Schaller/Erik Gawel/Klaas Korte
}

() Der/die Autor(en) 2021. Dieser Artikel ist eine Open-Access-Publikation.

\begin{abstract}
Der Weltklimarat IPCC zeigt in seinem Sonderbericht „1, $5^{\circ} \mathrm{C}$ globale Erwärmung" aus dem Jahr 2018, dass das Erreichen der im Pariser Übereinkommen vereinbarten Klimaziele nach wie vor möglich ist. Dies erfordere allerdings nicht nur einen grundlegenden Wandel unserer Lebens- und Wirtschaftsweise sowie die Reduktion von Treibhausgasemissionen auf Null (spätestens in der zweiten Hälfte dieses Jahrhunderts), sondern auch die dauerhafte Entnahme erheblicher Mengen von $\mathrm{CO}_{2}$ aus der Atmosphäre. Letzteres kann durch eine Reihe unterschiedlicher Verfahren und Technologien erreicht werden, sogenannte Negativemissionstechnologien (NETs). Im diesem Beitrag werden NETs im internationalen Klimaregime verortet. Insbesondere soll ihre potentielle Funktion als Senke im Sinne der Klimarahmenkonvention und des Pariser Übereinkommens untersucht werden.
\end{abstract}

\section{1. Überblick}

In dem bereits im vorherigen Heft erschienen Beitrag wurden die aktuell diskutierten NETs überblicksartig vorgestellt. ${ }^{1}$ Wirkungen und mögliche Nebenwirkungen wurden angesprochen. Weitergehend wurden ihre grundlegenden strukturellen Unterschiede zu den Maßnahmen der Begrenzung von Treibhausgasen, zur Anpassung sowie zum solaren Strahlungsmanagement (SRM) herausgearbeitet. Im diesem zweiten Teil gilt es, die verschiedenen Techniken im internationalen Klimaregime $\mathrm{zu}$ verorten (2). Insbesondere sol ihre potentielle Funktion als Senke im Sinne der Klimarahmenkonvention (KRK) und des Pariser Übereinkommens untersucht werden $(2.2,2.3)$. Hierzu erfolgt u.a. auch ein Rückblick auf die bisherige Praxis der Nutzung von Senken (2.4). Hieraus sollen einige Rückschlüsse hinsichtlich der zentralen Rechtsfragen gezogen werden, die sich im Zusammenhang mit einer möglichen Entwicklung und Anwendung von NETs als Instrumente der Klimapolitik stellen. AnschlieBend wird eine rechtliche und ökonomische Forschungsperspektive entwickelt (3). Der Beitrag endet mit einem Fazit (4). Untersuchungsleitend ist die These, dass die zunehmende Bedeutung von NETs als Instrument der Klimapolitik, ihre klare analytische, begriffliche und rechtsdogmatische Abgrenzung von anderen klimapolitischen Maßnahmen erfordert. Würden NETs in dem vom IPCC angedachten Umfang zum Einsatz kommen, ergäbe sich hieraus eine völlig neue Sachlage, die es im Klimaregime abzubilden gälte.

\section{NETs im internationalen Klimaregime}

Im folgenden Abschnitt gilt es überblicksartig zu klären, durch welche internationalen Regime die Erforschung und Anwendung von NETs derzeit geregelt ist bzw. geregelt werden könnte. Im Anschluss soll vertiefend untersucht werden, inwieweit NETs durch das internationale Klimaregime erfasst sind, ob und wie sie in dessen Rahmen bereits eingesetzt wurden und welche Rechtsfragen sich hieraus für ihre weitere Erforschung und potentielle Anwendung ableiten lassen.

PD Dr. Till Markus, LL.M., Dr. Romina Schaller, LL.M.Eur. Prof. Dr. Erik Gawel, Klaas Korte,

Department Umwelt- und Planungsrecht,

Helmholtz-Zentrum für Umweltforschung,

Leipzig, Deutschland
2.1 Vorüberlegungen zu den NETs im internationalen Recht und in den internationalen Beziehungen

Es gibt derzeit kein internationales Abkommen, das speziell die Erforschung oder die Anwendung von NETs umfassend regelt. Das ist bei neuen Technologien oder technischen Verfahren aber die Regel, d.h. sie entwickeln sich in bestehende Ordnungsvorstellungen und Regelungszusammenhänge ,hinein“, von denen sie zu Beginn noch unzureichend erfasst sind. ${ }^{2}$ Allerdings gibt es eine große Zahl von Rechtsakten auf internationaler Ebene, deren Anwendungsbereiche und Einzelnormen durch NETs berührt werden. ${ }^{3}$ Entsprechend der Vielgestaltigkeit der NETs betrifft das Übereinkommen aus den Bereichen des KlimaAtmosphären-, Biodiversitäts-, und Meeresumweltschutzes, aber u. U. auch des Chemikalien- und Wasserrechts. Weiterhin einschlägig können Verträge sein, die eher prozedurale Verpflichtungen für Staaten bereithalten (z.B. Kooperations- und Informationsverpflichtungen, UVPPflichten, Berichtspflichten etc.). Auch Abkommen über Haftungsfragen für potentiell durch NETs entstehende Schäden sind Teil des Rechtsrahmens, den es zu verstehen gilt, will man die rechtlichen Fragen erkennen, die sich im Zusammenhang mit der Entwicklung und Anwendung von NETs stellen. ${ }^{4}$ Dort wo Staaten NETs großskalig erforschen und implementieren, könnte außerdem in die Schutzbereiche international garantierter Menschenrechte eingegriffen werden. Denkbar wäre insoweit z.B., dass Vorgaben für die Nutzung von Wäldern die Eigentumsrechte von Waldbesitzern einschränken. ${ }^{5}$ Andererseits könnte die Regulierung der Durchführung wissenschaftlicher Experimente in die Forschungsfreiheit von Wissenschaftlern eingreifen. ${ }^{6}$

Aufgrund der Vielzahl möglicherweise einschlägiger Regime kommt es in der Praxis der internationalen Beziehungen zur Frage des ,forum choices“, d. h. unter welchem Vertragswerk soll eine NET (oder ein Aspekt davon) bei Bedarf geregelt werden? ${ }^{7}$ Die Beispiele der Ozeandüngung und des

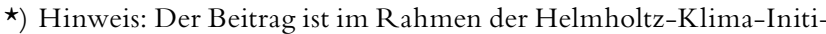
ative (HI-CAM) erarbeitet worden. HI-CAM wurde mit Mitteln des Impuls- und Vernetzungsfonds der Helmholtz-Gemeinschaft (IVF) gefördert. Die Verantwortung für den Inhalt dieser Veröffentlichung liegt bei der Autorin und den Autoren.

1) Markus/Schaller/Gawel/Korte, NuR 2020, 90 .

2) Siehe hierzu grundlegend Lin, Prometheus Reimagined: Technology, Environment, and Law in the Twenty-first Century, 2017; Nach wie vor lesenswert Däubler, ZRP 1986, 42.

3) Siehe Übersicht z. B. bei Reynolds, International Law, in: Gerrard/ Hester, Climate Engineering and the Law, 2018, S. 57; Krüger, Geoengineering und Völkerrecht, 2020.

4) Siehe insbesondere Hester, in: Gerrard/Hester, Climate Engineering and the Law, 2018, S. 224

5) Siehe z.B. Burns, in: Abate, Climate Justice: Case Studies in Global and Regional Governance Challenges, 2016, 149.

6) Hubert, The Human Right to Science and Its Relationship with International Environmental Law, EJIL 2020, i.E.

7) Armeni/Redgwell, International Legal and Regulatory Issues of Climate Geoengineering Goverance: Rethinking the Approach, 2015; Ginzky, in: Dilling/Markus, Ex Rerum Natura Ius - Recht aus der Natur der Sache, 2014, S. 105. Grundlegend auch Boyle/ Chinkin, The Making of International Law, 2007, S. 103-108, S. $144-151$. 
marinen CCS haben gezeigt, dass die Regelung einzelner NETs bzw. ihnen eng verbundener Technologien nicht zwingend im Rahmen des Klimaregimes erfolgen muss. ${ }^{8}$ Aufgrund der ihnen zugedachten Funktion als Instrumente der Klimaschutzpolitik kommt dem UN-Klimaregime für die rechtliche Steuerung der NETs aber quasi automatisch eine herausragende Bedeutung zu. Das Klimaregime setzt durch die Vorgabe verschiedener Ziele, Mechanismen und Verpflichtungen einen wesentlichen Anlass für ihre Erforschung und ihren möglichen Einsatz. Die folgenden $\mathrm{Ab}-$ schnitte sollen untersuchen, inwieweit NETs bereits durch das bestehende Klimaregime erfasst sind und welche normativen Vorgaben es für deren Erforschung und etwaige Anwendung enthält.

\subsection{NETs in der Klimarahmenkonvention}

Die Klimarahmenkonvention (KRK) ist nach wie vor das zentrale Abkommen, durch das die internationale Gemeinschaft ihre Bemühungen um den Schutz des Klimas koordiniert. Als Rahmenabkommen enthält sie vor allem wichtige Ziele, verschiedene Definitionen, Prinzipien, einen ersten Pflichtenkatalog sowie institutionelle und prozedurale Vorschriften. Konkretisierungen der in ihr enthaltenen Vorschriften erfolgten seit ihrem Inkrafttreten vor allem durch die Beschlüsse der Vertragsstaatenkonferenz sowie insbesondere durch das im Jahr 1997 beschlossene Kyoto-Protokoll (Inkrafttreten am 16. Februar 2005). Ihm folgend wurde Ende 2015 das bereits eingangs erwähnte PÜ unter der KRK vereinbart (Inkrafttreten am 4. November 2016). Im Folgenden gilt es zu bestimmen, ob NETs grundsätzlich in den Anwendungsbereich der KRK fallen, ob sie einen Beitrag zur Verwirklichung der in ihr festgelegten Ziele leisten können, ob die KRK ihnen einen bestimmten Rang in der Liste der Klimaschutzinstrumente zuweist und wie sie im Lichte des in Art. 3 Abs. 3 der KRK festgelegten Vorsorgeansatzes zu bewerten sind. Im Rahmen der Prüfung sollen die oben eingeführten Unterscheidungen zwischen den Maßnahmen zur Vermeidung der Bildung von THGs, ihrer Zurückhaltung, ihrer Entnahme, der Anpassung und des SRM berücksichtigt werden.

\subsubsection{Anwendungsbereich}

Der Anwendungsbereich der KRK ist weit gefasst. Ihre Vorschriften beziehen sich auf die zentralen Aspekte der Klimaproblematik. Umfasst sind die Themen der Abschwächung (Mitigation), der Anpassung, der Erforschung, des Technologie- und Finanztransfers, der Bildung und Bewusstseinsschaffung sowie Mechanismen zur Beilegung von Streitigkeiten. Die KRK war aber von Beginn an entwicklungsoffen angelegt, d.h. die Weiterentwicklung des international-koordinierten Klimaschutzes sollte in ihrem Rahmen durch Protokolle und Vertragsstaatenbeschlüsse erfolgen. ${ }^{9}$ Während der Vertragsverhandlungen waren tatsächlich zwei Fragen strittig, nämlich ob sich das Abkommen lediglich auf das Treibhausgas $\mathrm{CO}_{2}$ beschränken und ob das Konzept der Senken aufgenommen werden solle. Beides wurde im Ergebnis bejaht, weshalb man auch von einem comprehensive approach spricht. Letzterer besagt, dass Maßnahmen zur Abschwächung (Mitigation) der Klimaänderung sowohl Quellen von als auch Senken für Treibhausgasen umfassen solle. ${ }^{10}$ Entscheidend ist an dieser Stelle, dass der Anwendungsbereich der KRK weit gefasst ist und sie grundsätzlich die Entnahme von Treibhausgasen als Instrument der Klimapolitik vorsieht. Unter anderem indiziert die Definition der Speicher (,Bestandteile des Klimasystems, in denen ein Treibhausgas (...) zurückgehalten wird“") ${ }^{11}$, dass es sich bei den Senken ursprünglich vor allem um natürliche Verfahren handeln sollte, die „Bestandteile des Klimasystems“ sind, also Land, Biomasse und Meere. ${ }^{12}$ Darauf deutet auch die Entstehungs- und Verhandlungsgeschichte der Senkenregeln hin, bei der vor allem Maßnahmen der verbesserten Landnutzung sowie forstwirtschaftliche Maßnahmen diskutiert wurden. ${ }^{13}$ Nicht zuletzt hebt Art. 4 Abs. 1 lit. d) KRK hinsichtlich der Erhaltung und Verbesserung von Senken insbesondere die „Biomasse, Wälder, und Meere sowie andere Ökosysteme auf dem Land, an der Küste und im Meer" hervor.

\subsubsection{Problem, Ursachen und Folgen des Klimawandels}

Oben wurde grundlegend zwischen Maßnahmen unterschieden, die sich auf die Vermeidung der Entstehung, die Zurückhaltung, und die Entnahme von Treibhausgasen beziehen. Daneben stehen die Maßnahmen der Anpassung und des Strahlungsmanagements. Diese analytische Trennung entspricht den Regelungen der KRK. Auch diese unterscheidet zwischen der Bildung von Treibhausgasen, ihrer Übergabe an die Atmosphäre, ihrer Akkumulation in der Atmosphäre und der sich daraus ergebenden Erwärmung der Erde.

So definiert Art. 1 Abs. 2 Klimaänderungen als „Änderungen des Klimas, die unmittelbar oder mittelbar auf menschliche Tätigkeiten zurückzuführen sind, welche die Zusammensetzung der Erdatmosphäre verändern (...)." Hier wird die Entstehung der Treibhausgase durch menschliches Tun angesprochen. Wie oben bereits erläutert definiert Art. 1 Nr. 9 KRK ,Quelle“ als Vorgang oder Tätigkeit, „,durch die ein Treibhausgas (...) in die Atmosphäre freigesetzt" wird. Die Akkumulation der Treibhausgase in der Atmosphäre als Folge der anthropogenen Verursachung und der Freisetzung wird dann als die zentrale Herausforderung des Klimaproblems bestimmt, die es gem. Art. 2 KRK auf einem Niveau zu stabilisieren gilt, auf dem eine gefährliche anthropogene Störung des Klimasystems verhindert wird. Art. 1 Abs. 1 KRK beschreibt wiederum die Folgen des Problems der sich akkumulierenden Treibhausgaskonzentration als ,nachteilige Auswirkung der Klimaänderungen.“ Dies seien die ,sich aus den Klimaänderungen ergebenden Veränderungen der belebten oder unbelebten Umwelt (...)“.

\subsubsection{Ziele der Klimarahmenkonvention}

Art. 2 KRK enthält das zentrale Ziel des Klimaregimes sowie drei weitere Schutzziele, die es bei der Verfolgung des ersten Ziels zu berücksichtigen gilt. Gleichzeitig gibt er einen Zeitrahmen vor, innerhalb dessen dieses Ziel erreicht werden soll.

Das Endziel dieses Übereinkommens und aller damit zusammenhängenden Rechtsinstrumente ..., ist es, ... die Stabilisierung der Treibhausgaskonzentrationen in der Atmosphäre auf einem Niveau zu erreichen, auf dem eine gefährliche anthropogene Störung des Klimasystems ver-

8) Die Forschung ist recht umfangreich geregelt, die kommerzielle Nutzung ist derzeit noch verboten. Siehe hierzu eingehend Ginzky, in: Salomon/Markus, Handbook on Marine Environment Protection, 2018, S. 997; Ginzky/Frost, Marine Geo-Engineering: Legally Binding Regulation under the London Protocol, CCLR 2014, 82; Güssow, Sekundärer maritimer Klimaschutz: Das Beispiel der Ozeandüngung, 2012. Siehe hierzu umfassend die Informationen auf der Website der International Maritime Organisation (IMO): abrufbar unter http://www.imo.org/en/ OurWork/Environment/LCLP/EmergingIssues/CCS.

9) Art. $17 \mathrm{KRK}$.

10) Bodansky/Brunnée/Rajamani, International climate change law, 2017, S. 133.

11) Art. $1 \mathrm{Nr} .7 \mathrm{KRK}$

12) Bestandteile des Klimasystems sind neben der Atmosphäre, die Biosphäre, die Lithosphäre und die Hydrosphäre. Siehe hierzu erläuternd Simonis, in: ders., Handbuch Globale Klimapolitik, 2017, S. $48 \mathrm{f}$.

13) Gillespie, Sinks and the Climate Change Regime: The State of Play, Duke Environ. Law Policy Forum 2003, 279. 
hindert wird. Ein solches Niveau sollte innerhalb eines Zeitraums erreicht werden, der ausreicht, damit sich die Ökosysteme auf natürliche Weise den Klimaänderungen anpassen können, die Nahrungsmittelerzeugung nicht bedroht wird und die wirtschaftliche Entwicklung auf nachhaltige Weise fortgeführt werden kann.

Aus den hier festgelegten Zielen und der Funktionsweise der NETs wurde richtigerweise eine Priorisierung der Maßnahmen zur Vermeidung der Entstehung von Treibhausgasen gegenüber Maßnahmen der Rückhaltung und Entnahme abgeleitet. ${ }^{14}$ Eine derartige Lesart folgt aus der besonderen Geeignetheit der ersten Maßnahmengruppe als Mittel zur Erreichung einer dauerhaften Stabilisierung der Treibhausgaskonzentration in der Atmosphäre. Probleme hinsichtlich der langfristigen Einlagerung stellen sich bei vermiedenen - anders als bei nach ihrer Entstehung zurückgehaltenen oder entnommenen - Treibhausgasen nicht. ${ }^{15}$ Weitergehend betont die KRK hier in Art. 2 sowie an mehreren weiteren anderen Stellen ${ }^{16}$, dass Klimaschutz auch Umwelt- und soziale Belange zu berücksichtigen habe. Vor dem Hintergrund der derzeitigen Erkenntnisse über die ökologische Bilanz von Maßnahmen zur Vermeidung der Entstehung von Treibhausgasen einerseits und derjenigen der Rückhaltung und Entnahme andererseits, heben sich erstere gegenüber letzteren positiv ab. ${ }^{17}$ Das mag perspektivisch allerdings nicht für alle NETs gelten.

\subsubsection{Vorsorge}

Das Vorsorgeprinzip war Gegenstand umfangreicher Diskussionen im Kontext des Climate Engineering. ${ }^{18}$ Aufgrund der strukturellen Ähnlichkeiten sowie Überschneidungen einzelner NETs mit diesen Ansätzen werden die damals diskutierten Fragen mit großer Sicherheit auch für die zu erwartenden Debatten um NETs relevant werden. Dementsprechend soll hier auf seine Bedeutung für die Erforschung und den potentiellen Einsatz von NETs eingegangen werden.

Augenfällig war in der Diskussion um die Funktion des Vorsorgeprinzips in Bezug auf das Climate Engineering, dass es sowohl für als auch gegen die Erforschung einzelner Techniken in Stellung gebracht wurde. ${ }^{19}$ Nach der hier vertretenen Auffassung unterscheidet ein derartiges Verständnis nicht ausreichend trennscharf zwischen a) der anthropogen verursachten Entstehung, b) der Freisetzung und c) der Akkumulation der Treibhausgase in der Atmosphäre sowie d) der Erwärmung der Erde infolge der erhöhten atmosphärischen Treibhausgaskonzentration.

Was folgt nun aus dieser analytischen Teilung im Hinblick auf die Ausdeutung des Vorsorgeansatzes bezogen auf NETs?

Es gilt zuerst festzuhalten, dass viel über den Inhalt, die Normstruktur und den rechtlichen Status des Vorsorgeansatzes geschrieben und gestritten wurde. ${ }^{20}$ Im Ergebnis fehlt es immer noch an einem bereichsübergreifenden Verständnis. ${ }^{21}$ An dieser Stelle wird der Analyse die spezifische Variante des Vorsorgeansatzes in Art. 3 Abs. 3 KRK zugrunde gelegt. Aufgrund des möglichen Einsatzes von NETs als Instrumente des Klimaschutzes kommt ihm für deren Bewertung eine bedeutende Orientierungsfunktion zu. ${ }^{22}$ In Art. 3 Abs. 3 KRK steht:

Die Vertragsparteien sollen Vorsorgemaßnahmen treffen, um den Ursachen der Klimaänderungen vorzubeugen, sie $z u$ verhindern oder so gering wie möglich zu halten und die nachteiligen Auswirkungen der Klimaänderungen abzuschwächen. In Fällen, in denen ernsthafte oder nicht wiedergutzumachende Schäden drohen, soll das Fehlen einer völligen wissenschaftlichen Gewissheit nicht als Grund für das Aufschieben solcher Maßnahmen dienen, wobei zu berücksichtigen ist, dass Politiken und Maßnahmen zur Bewältigung der Klimaänderungen kostengünstig sein sollten, um weltweite Vorteile zu möglichst geringen Kosten zu gewährleisten. Zur Erreichung dieses Zwecks sollen die Politiken und Maßnahmen (...) alle wichtigen Quellen, Senken und Speicher von Treibhausgasen und die Anpassungsmaßnahmen erfassen sowie alle Wirtschaftsbereiche einschließen. Bemühungen zur Bewältigung der Klimaänderungen können von interessierten Vertragsparteien gemeinsam unternommen werden. (Hervorhebungen durch die Autoren).

Grundsätzlich wird erkennbar, dass das Vorsorgeprinzip auf eine Ungewissheitssituation reagiert. Prima facie ist Art. 3 Nr. 3 KRK hinsichtlich NETs somit recht offen formuliert. Der erste Satz zu den Vorsorgemaßnahmen verlangt, dass diese ergriffen werden sollen, um den Ursachen der Klimaänderungen vorzubeugen, sie zu verhindern oder so gering wie möglich zu halten. Den Ursachen vorzubeugen bedeutet im Lichte des Art. 1 Abs. 2 KRK, Maßnahmen zu ergreifen, die die ,menschlichen Aktivitäten“ adressieren, ,welche die $\mathrm{Zu}$ sammensetzung der Erdatmosphäre verändern." Der zweite Teil des ersten Satzes bezieht auch Maßnahmen der Anpassung mit ein, die dem Zweck der Abschwächung der „nachteiligen Auswirkungen der Klimaänderungen“ dienen. ${ }^{23}$ Das auf beide Arten von Maßnahmen bezogene Vorsorgeprinzip in Art. 3 Abs. 3, S. 2 KRK erkennt dann an, dass die entsprechenden Politiken und Maßnahmen ,,alle wichtigen Quellen, Senken und Speicher von Treibhausgasen erfassen." Weitergehend umfasst der Wortlaut des letzten Satzes des Art. 3 Nr. 3 KRK recht offen die „Bewältigung der Klimaänderungen“. Insoweit spricht sich Art. 3 Nr. 3 KRK nicht eindeutig gegen das vorsorgliche Ergreifen von Maßnahmen aus, die auf die Entnahme von Treibhausgasen aus der Atmosphäre gerichtet sind, was somit grundsätzlich auch einige NETs einschließen könnte.

Art. 3 Abs. 3 KRK fordert also in erster Linie das Ergreifen von Maßnahmen, die die Entstehung von anthropogenen Treibhausgasen adressieren. Auch Maßnahmen der Anpassung sind zu ergreifen, also Maßnahmen die den Umgang mit der Erwärmung der Erde als Folge der anthropogenen Verursachung von Treibhausgasen bezwecken. Darüber hinaus zeigt sich der in Art. 3 Abs. 3 KRK enthaltene Ansatz offen für den vorsorglichen Einsatz von Senken (insbesondere solcher, die Teil des Klimasystems sind), nicht zuletzt auch, um Kostenerwägungen $\mathrm{zu}$ ermöglichen.

Im Lichte der aus Art. 2 der KRK abgeleiteten Priorisierung von Maßnahmen zur Vermeidung der Entstehung

14) So auch Stoll/Krïger, in: Proelß, Internationales Umweltrecht, S. 281, Rdnr. 12-14.

15) Stoll/Krüger, in: Proelß, Internationales Umweltrecht, S. 281 Rdnr. 12-14. So auch Güssow, Sekundärer maritimer Klimaschutz: Das Beispiel der Ozeandüngung, 2012, S. $61 \mathrm{f}$.

16) Abschnitte 2, 7, 8, 10, 12, 17, 19 der Präambel, sowie Art. 1 Nr. 1 und Art. 4 Abs. 1 lit. f) KRK.

17) Ähnlich Craik/Burns, Climate Engineering under the Paris Agreement: A Legal and Policy Primer, Centre for International Governance Innovation, 2016, S. 7 f.

18) Siehe Überblick bei Krüger, Geoengineering und Völkerrecht, 2020, S. $409 \mathrm{f}$.

19) Hierzu auch Proel $\beta$, JZ 2011, 495, 498

20) Im Klimazusammenhang zuletzt Hartzell-Nichols, A Climate of Risk - Precautionary Principles, Catastrophes, and Climate Change, 2017. Siehe auch die Diskussion bei Proelß, JZ 2011, 495, 498; Stoll, in: Dilling/Markus, Ex Rerum Natura Ius? Sachzwang und Problemwahrnehmung im Umweltrecht, 2014, S. 37.

21) Proelß, JZ 2011, 495, 498.

22) Der Eröffnungssatz des Art. 3 KRK besagt, dass sich die Vertragsparteien von den folgenden Prinzipien „leiten“ lassen. Durch die Wortwahl sollte der Grad der Verbindlichkeit gegenüber regulären Rechtsprinzipien reduziert werden. Bodansky/Brunneée/Rajamani, International climate change law, 2017, S. 133S. 126-127.

23) Stoll/Krüger, in: Proelß, Internationales Umweltrecht, S. 281, Rdnr. 12-14. 
von Treibhausgasen gegenüber solchen der Rückhaltung und Entnahme, sind erstere auch bei der Anwendung des Vorsorgeansatzes zu priorisieren. Auch Art. 3 Abs. 3 KRK nennt die Maßnahmen zur Vorbeugung der „Ursachen“ zuerst. Hinzu kommt, dass Art. 3 Abs. 3 KRK vorsorgliche Maßnahmen zur Anpassung und Entnahme zwar nicht ausschließt, die Herleitung eines Gebots oder der Legitimation der Erforschung oder Anwendung von NETs im Hinblick auf den Telos des Vorsorgeansatzes insgesamt problematisch erscheint. Dort wo NETs emittierte Treibhausgase durch den Einsatz zusätzlicher Energie oder zusätzlichen Materials klimaunwirksam machen, adressieren sie im Verständnis der KRK nicht die Ursachen der Klimaänderungen, sondern lediglich das Problem der erhöhten Treibhausgaskonzentration in der Atmosphäre (Akkumulation). Forderte man die Erforschung und ggf. den Einsatz von NETs auf der Grundlage des Vorsorgeansatzes, würde man also ,vorsorgliche Nachsorge“ verlangen.

Die hier angestellten Uberlegungen beseitigen indes nicht das Problem, dass es zu entscheiden gilt, ob durch die Erforschung oder Anwendung von NETs erzeugte Risiken hinzunehmen sind, um so ggf. noch größere durch den Klimawandel erzeugte Gefahren und Schäden abzuwenden. Zur Auflösung dieses Dilemmas mögen verschiedene rechtliche Grundlagen angeführt oder neu entwickelt werden, der Vorsorgeansatz eignet sich hierfür nicht. ${ }^{24}$ Das gilt insbesondere für die Fälle, in denen NETs erhebliche Schäden im Hinblick auf andere Umweltgüter befürchten lassen. ${ }^{25}$ Sollten die Parteien die Erforschung und Anwendung von NETs weiterverfolgen, gebietet das Vorsorgeprinzip vielmehr das proaktive Ergreifen von Maßnahmen zum Schutz der Umwelt vor den sich daraus potentiell ergebenden Gefahren.

\subsection{NETs im Pariser Übereinkommen}

Wie die KRK nennt auch das Pariser Übereinkommen (PÜ) NETs nicht ausdrücklich und wie die KRK sieht es die Verwendung von Senken als Klimaschutzmaßnahmen vor. ${ }^{26}$ Insbesondere fordert das PÜ die Vertragsparteien auf, zur Erreichung des langfristigen Temperaturziels ,,in der zweiten Hälfte dieses Jahrhunderts ein Gleichgewicht zwischen den anthropogenen Emissionen von Treibhausgasen aus Quellen und dem Abbau solcher Gase durch Senken ... „herzustellen. ${ }^{27}$ Daneben sollen die Vertragsparteien verminderte ,anthropogene Emissionen“ und den „Abbau“ von Treibhausgasen auch im Hinblick auf die Erreichung ihrer NDCs berücksichtigen. ${ }^{28}$ In diesem Zusammenhang ist an die oben genannten Staaten zu erinnern, die vor kurzer Zeit verschiedene NETs als Instrumente zur Erreichung ihrer nationalen Klimaziele benannt haben. Art. 5 Abs. 1 des PÜ hebt hinsichtlich der Verpflichtung der Vertragsparteien, Maßnahmen zur Erhaltung und Verbesserung von Senken zu ergreifen, „Wälder“ als einzige Maßnahme ausdrücklich hervor. ${ }^{29}$ Weitere Aussagen zu Senken trifft das PÜ nicht.

\subsection{Bisherige und zukünftige Relevanz von NETs im Klimaregime}

Was folgt nun aus den allgemeinen Vorschriften der beiden zentralen Vertragswerke des internationalen Klimaschutzrechts im Hinblick auf NETs? In einem ersten Schritt ist zu klären, in welchem Umfang und unter welchen Bedingungen die Entnahme von Treibhausgasen aus der Atmosphäre durch Senken im Rahmen der beiden Abkommen bisher erfolgt ist. Vor dem Hintergrund dieser Erfahrungen soll in einem zweiten Schritt geschlossen werden, wo wichtige rechtliche und ökonomische Fragen für eine mögliche Integration von NETs in das Klimaregime liegen könnten.

Zur Implementierung der Senkenfunktion waren vor allem landnutzungs- oder waldbezogene Maßnahmen sowie das CCS vorgesehen. ${ }^{30}$ Von praktischer Bedeutung waren bisher aber vor allem die landnutzungs- und waldbezogenen Maßnahmen. Diese erfolgten im Wesentlichen in drei Zusammenhängen: Als Maßnahmen entwickelter Staaten (sog. „Annex-I-Staaten“) zur Erreichung ihrer verbindlichen Emissionsreduktionsziele unter dem Kyoto-Proto$\mathrm{koll}^{31}$, zur Generierung von Carbon Removal Units im Zuge der Umsetzung des Joint Implementation und des Clean Development Mechanisms (ebenfalls im Rahmen des Kyoto-Protokolls), ${ }^{32}$ im Gegenzug für finanzielle oder anderweitige Unterstützung für reduzierte Emissionen aus der Entwaldung oder Waldschädigung in Entwicklungsländern (zuerst RED, dann REDD, dann REDD+). ${ }^{33}$ Die Entwicklung dieser Ansätze hat sich allerdings über gut zwei Jahrzehnte hingezogen, war mit erheblichen Herausforderungen verbunden und die kritischen Fragen hinsichtlich ihrer Effektivität sind bis heute nicht ganz verstummt. ${ }^{34}$

Im Rahmen des Kyoto-Protokolls waren die entwickelten Staaten verpflichtet, Emissionsreduktionen vorzunehmen. Bei der Berechnung der reduzierten Mengen konnten die sogenannten ,Land Use and Land-Use Change and Forestry" (LULUCF) bezogenen Maßnahmen berücksichtigt werden. ${ }^{35}$ Den Vertragsparteien war es möglich, zwischen verschiedenen Maßnahmen zu wählen (revegetation, cropland management, grazingland management, wetland drainage and rewetting, forest management). War die Treibhausgasbilanz der Senken negativ, erhielt die jeweilige Partei die sogenannten Removal Units (RMUs). Die Möglichkeiten

24) Siehe zur Problematik auch Proelß, (Fn. 19).

25) Gegen die Berufung auf das Vorsorgeprinzip zur Erforschung und ggf. Anwendung von NETs spricht indes nichts, solange derartige Maßnahmen selbst keine Gefahren für die Umwelt erzeugen oder sogar noch Synergieeffekte mit anderen Umweltzielen verwirklichen.

26) Reynolds, International Law, in: Gerrard/Hester, Climate Engineering and the Law, 2018, S. 69.

27) Art. 4 Abs. 1 PÜ.

28) Art. 4 Abs. 13 und 14 PÜ.

29) In Art. 5 Abs. 2 PÜ wird auch die Bedeutung der waldbezogenen Maßnahmen des REDD+ Mechanismus hervorgehoben.

30) Siehe Art. 3 Abs. 3 und Abs. 4 Kyoto-Protokoll. Siehe auch die Entscheidung der Parteien des Kyoto-Protokolls, CCS als zulässiges Instrument zur Erreichung der Reduktionsziele anzuerkennen, UNFCCC, Report of the Conference of the Parties serving as the meeting of the Parties to the Kyoto Protocol on its seventh session, held in Durban from 28 November to 11 December 2011 (FCCC/KP/CMP/2011/10/Add.2), Decision 10/ CMP.7. Siehe hierzu insbesondere Redgwell, International Legal Responses to the Challenges of a Lower-Carbon Future: Climate Change, Carbon Capture and Storage, and Biofuels, in: Zillman et al., Beyond the Carbon Economy, 2008, S. 85, $94 \mathrm{f}$.

31) Siehe Art. 3 Abs. 3 und 4 Kyoto-Protokoll.

32) Siehe Art. 6 und 12 Kyoto-Protokoll.

33) REDD+ steht für Reduktion von Emissionen aus Entwaldung und Schädigung der Wälder plus Erhaltung von Kohlenstoffvorräte im Wald, der nachhaltigen Waldbewirtschaftung und der Erhöhung der Kohlenstoffbestände von Wäldern in Entwicklungsländer. Siehe UNFCCC, Report of the Conference of the Parties on its sixteenth session, held in Cancun from 29 November to 10 December 2010 (FCCC/CP/2010/7/Add.1), Decision 1/CP.16.

34) Senken waren eben von Beginn an problematische Elemente des Klimaregimes, siehe im Rückblick Gillespie, Sinks and the Climate Change Regime: The State of Play, Duke Environ. Law Policy Forum 2003, 279. Siehe auch Grassi et al., The Key Role of Forests in Meeting Climate Targets Requires Science for Credible Mitigation, Nat. Clim. Chang. 2017, 220. Siehe auch FAO, Forestry for a Low Carbon Future, 2016.

35) Siehe Art. 3 Abs. 3 und Abs. 4 Kyoto-Protokoll. Die Voraussetzungen wurden näher ausgeführt in UNFCCC, Report of the Conference of the Parties serving as the meeting of the Parties to the Kyoto Protocol on its seventh session, held in Durban from 28 November to 11 December 2011 (FCCC/KP/CMP/2011/10/ Add.1), Decision 2/CMP.7. 
zur Anrechnung der RMUs auf die Reduktionsverpflichtungen waren allerdings begrenzt. ${ }^{36}$ Weiterhin konnten entwickelte Staaten durch den Joint Implementation und den Clean Development Mechanism Klimaschutzmaßnahmen in anderen Ländern durchführen, dadurch Emission Reduction Units bzw. Certified Emission Reductions erwerben und so ihren Reduktionsverpflichtungen nachkommen. ${ }^{37}$ Alle durch Senken erworbenen Units konnten im Rahmen des internationalen Emissionshandels eingesetzt werden. ${ }^{38}$ In diesem Zusammenhang ist allerdings bedeutsam, dass für Senkenprojekte nur spezielle zeitlich beschränkte Reduction Units vergeben wurden (temporary certified emission reductions/long-term certified emission reductions). Am Ende ihrer Laufzeit waren sie zu ersetzen. Der Grund dafür lag in der zeitlich begrenzten Bindung der Treibhausgase. ${ }^{39}$ Stoll und Krüger weisen mit Recht darauf hin, dass sich in der Beschränkung der Einsatzmöglichkeiten von Senken zur Erfüllung der Treibhausgasreduktionsverpflichtungen sowie im Rahmen der flexiblen Mechanismen unter dem Kyoto-Protokoll die bereits in der KRK angelegte Priorisierung der Instrumente erneut zeigt. ${ }^{40}$ LULUCF-Maßnahmen haben in der Praxis beider Mechanismen eine eindeutig untergeordnete Rolle gespielt. Gründe hierfür waren einerseits ihre hohen Transaktionskosten, die mengenmäßige Beschränkung der Senkenprojekte sowie ihre eingeschränkte Anrechenbarkeit auf die nationalen Emissionsreduktionsverpflichtungen sowie im Rahmen der flexiblen Mechanismen. ${ }^{41}$

Bei REDD+ geht es darum, dass Industrieländer (oder andere Geber aus den industrialisierten Staaten ${ }^{42}$ ) im Wege verschiedener Finanzierungsformen Entwicklungs- und Schwellenländer dafür kompensieren, dass diese ihre Wälder schützen und dadurch Emissionen aus Entwaldung und Walddegradierung vermeiden. Allerdings erfolgt die entsprechende Kompensation lediglich unter bestimmten Voraussetzungen. ${ }^{43}$ Insbesondere müssen die Entwicklungsund Schwellenländer eine nationale Strategie oder einen Aktionsplan erstellen, ein nationales Referenzniveau (Bezugspunkt) für die Messung der Emissionsreduzierungen bestimmen, ein Überwachungssystem zur Verifizierung und Berichterstattung der erzielten Emissionsreduktionen schaffen sowie ein Safeguards-Informationssystem etablieren. Letzteres dient der Verwirklichung der in Anhang I des Beschlusses 1/CP.16 festgelegten Klauseln ${ }^{44}$ zum Schutz indigener bzw. lokaler Bevölkerung sowie der biologischen Vielfalt. ${ }^{45}$ Die Etablierung des REDD+-Mechanismus zog sich über gut ein Jahrzehnt hin. Insbesondere die Festlegung der Bewertungskriterien für die waldbezogenen Maßnahmen sowie die Finanzierung der Projekte gestaltete sich schwierig. Ein Großteil der REDD+-basierten Förderung ist daher auch bisher vor allem zur Schaffung der institutionellen Voraussetzungen der eigentlichen Maßnahmen verwendet worden, d. h. zur Entwicklung der nationalen Strategien, für das capacity-buidling sowie zur Stärkung der zuständigen nationalen Behörden.

Gesetzt den Fall, dass einzelne NETs eines Tages als geeignete Mittel des Klimaschutzes eingesetzt würden, wäre auf jeden Fall ihre Funktion als Senke im Rahmen des Klimaregimes zu klären. Das ist letztlich auch unabhängig von der Frage, in welchem Regime einzelne NETs geregelt werden. Sollte z. B. die Regelung meeresbasierter NETs im Rahmen der ,Convention on the Prevention of Marine Pollution by Dumping of Wastes and Other Matter" (London Convention and Protocol) erfolgen, müssten etwaige Treibhausgasreduktionen dennoch im Rahmen des Klimaregimes anerkannt werden, z.B. für ihre Anrechenbarkeit auf die NDCs der Vertragsparteien oder im Hinblick auf ihre Integration in den im Rahmen des Art. 6 Abs. 2 PÜ noch zu entwickelnden ,kooperativen Ansatz“, der die „Verwendung international übertragbarer Minderungsergebnisse zum Erreichen der national festgelegten Beiträge" beinhaltet. Es geht also im Kern um die Möglichkeit der Verrechenbarkeit der durch NETs aus der Atmosphäre entnommenen und eingelagerten Treibhausgase und ihre Integration in einen internationalen Emissionshandel oder dem Joint Implementation oder CDM nachfolgende Instrumente. Wesentlich ist insoweit vor dem Hintergrund der bisherigen Erfahrungen mit Senken im Rahmen des Klimaregimes die Festlegung von klaren Methoden, Kriterien und Verfahren, mit denen die Entnahme von Treibhausgasen quantifiziert und möglichst eindeutig nachvollzogen werden kann. Das dürfte bei technologischen Ansätzen leichter sein als bei naturbasierten Verfahren. ${ }^{46}$ Im Hinblick auf den Einsatz naturbasierter Verfahren lässt sich insbesondere aus den Erfahrungen im Zusammenhang mit REDD+ lernen, dass Mechanismen geschaffen werden müssen, die nachteilige soziale und ökologische Nebenwirkungen land- oder waldbezogener Projekte wirksam verhindern. ${ }^{47}$

\section{Forschungsperspektiven}

Die Erörterung der Forschungsperspektiven beginnt mit einem Dilemma: Sollen Zeit und Geld investiert werden, um in die Erforschung und ggf. Entwicklung von Verfahren und Technologien zu investieren, deren Beitrag zur Lö-

36) Siehe UNFCCC, Report of the Conference of the Parties serving as the meeting of the Parties to the Kyoto Protocol on its first session, held at Montreal from 28 November to 10 December 2005 (FCCC/KP/CMP/2005/8/Add.3), Decision 16/CMP.1, Annex, Para. $11 \mathrm{f}$; UNFCCC, Report of the Conference of the Parties serving as the meeting of the Parties to the Kyoto Protocol on its seventh session, held in Durban from 28 November to 11 December 2011 (FCCC/KP/CMP/2011/10/Add.1), Decision 2/CMP.7, Annex, Para. 13.

37) Art. 6 Abs. 1 lit. b) und Art. 12 Abs. 3. Kyoto-Protokoll

38) Siehe Art. 17 Kyoto-Protokoll. Hierzu Stoll/Krüger, in: Proelß, Internationales Umweltrecht, S. 312.

39) Stoll/Krüger, in: Proelß, Internationales Umweltrecht, S. 311

40) Stoll/Krüger, in: Proelß, Internationales Umweltrecht, S. 309.

41) Stoll/Krüger, in: Proelß, Internationales Umweltrecht, S. 310312. Siehe auch Lin, Prometheus Reimagined: Technology, Environment, and Law in the Twenty-first Century, 2017, S. 552.

42) Siehe hierzu auch UNFCCC, Report of the Conference of the Parties serving as the meeting of the Parties to the Kyoto Protocol on its seventh session, held in Durban from 28 November to 11 December 2011 (FCCC/CP/2011/9/Add.1), Decision 2/CP.17, Nr. 66.

43) UNFCCC, Report of the Conference of the Parties on its nineteenth session, held in Warsaw from 11 to 23 November 2013 (FCCC/CP/2013/10/Add. 1), Decision 9/CP.19, Nr. 1 i. V.m. UNFCCC, Report of the Conference of the Parties serving as the meeting of the Parties to the Kyoto Protocol on its seventh session, held in Durban from 28 November to 11 December 2011 (FCCC/CP/2011/9/Add.1), Decision 2/CP.17, Nr. 65. Schaller, Klimaschutz durch Walderhalt am Beispiel des REDD+-Mechanismus, 2020, S. 85.

44) Siehe UNFCCC, Report of the Conference of the Parties serving as the meeting of the Parties to the Kyoto Protocol on its first session, held at Montreal from 28 November to 10 December 2005 (FCCC/KP/CMP/2005/8/Add.3), Decision 1/CP.16.

45) Siehe die Warschauer Rahmenbedingungen zu REDD+. Diese enthalten insgesamt sieben Beschlüsse und stellen somit Politikansätze und einen umfassenden Leitfaden mit Elementen und Standards für die Implementierung des REDD+ in den Entwicklungsländern zur Verfügung. Siehe UNFCCC, Report of the Conference of the Parties on its nineteenth session, held in Warsaw from 11 to 23 November 2013 (FCCC/CP/2013/10/ Add. 1), Decision 9/CP.19-15/CP.19. Mehr dazu: Schaller, Klimaschutz durch Walderhalt am Beispiel des REDD+-Mechanismus, 2020, S. 90f; Voigt/Ferreira, The Warsaw Framework for REDD+: Implications for national implementation and access to results-based finance, CCLR 2015, 113, 120.

46) So auch Reynolds, International Law, in: Gerrard/Hester, Climate Engineering and the Law, 2018, S. 69.

47) Schaller, Klimaschutz durch Walderhalt am Beispiel des REDD+Mechanismus, 2020, S. $122 \mathrm{f} ., 73 \mathrm{ff}$. 
sung des Klimaproblems - soweit derzeit erkennbar - weitgehend unsicher ist (nicht zuletzt weil dies das Problem der dauerhaften Einlagerung erzeugen), die beträchtliche Risiken beinhalten und die eine Situation des ,,moral hazards" begründen?

Die Beantwortung dieser Frage könnte wegweisend sein für die staatliche Forschungsförderung. Es muss entschieden werden, welche Ansätze und Technologien mit den begrenzt zur Verfügung stehenden öffentlichen Mittel weiter erforscht werden sollen. Hierdurch wird nicht unwesentlich mitentschieden, ob bestimmte NETs weiterentwickelt werden oder nicht. Bereits auf dieser Ebene besteht weiterer Klärungsbedarf, sowohl in politischer als auch in rechtlicher Hinsicht. ${ }^{48}$

Viele Argumente sprechen durchaus für die weitere wissenschaftliche Erforschung der Funktionsweisen und Entnahmepotenziale von NETs. Vor diesem Hintergrund würde es nun gelten, einzelne, vielversprechende Maßnahmen mit größerer Tiefenschärfe zu untersuchen. Nur so können letztlich rationale Abwägungsentscheidungen hinsichtlich der Bedingungen ihres möglichen Einsatzes getroffen werden. Untersucht werden müssen sowohl technische als auch soziale, ökonomische, ökologische und rechtliche Fragen. Der verstärkte Einsatz von NETs durch Länder wie Kanada, Schweden und das Vereinigten Königreich bietet Gelegenheiten für empirische Untersuchungen. Diese können wertvolle Hinweise für die weiteren Perspektiven von NETs in Deutschland und auf europäischer Ebene geben.

Klärungsbedürftig werden auch die Bedingungen der Integration von NETs in nationale und internationale Emissionshandelssysteme sein. Hiervon hängt ganz wesentlich ab, ob die positiven Gestaltungspotenziale des Marktes angeregt werden können. Die bisherigen Erfahrungen mit Senken im Klimaregime zeigen, dass es hierzu objektiver und transparenter Berechnungsmethoden bedarf. Insbesondere gilt es, die begrenzte Dauerhaftigkeit der Einlagerungspotenziale von LULUCF- oder DACCU-Maßnahmen zu berücksichtigen.

Weiterhin gilt es, die Bedeutung der einzelnen NETs für die Klimapolitik der Europäischen Union sowie Deutschlands zu klären. ${ }^{49}$ Welche Rolle könnten sie im Rahmen des aktuell auf den Weg gebrachten europäischen Klimagesetzes spielen, welche Rolle kommt ihnen im deutschen Klimaschutzrecht potenziell zu? Damit ist auch die Frage der ,,richtigen“ Gewichtung in einem klimapolitischen Mix aus Mitigation, NETs und Anpassung angesprochen.

\section{Fazit und Ausblick}

Im Dezember 2019 zeichnete sich ab, dass es trotz der NDCs aller Vertragsparteien des PÜ in besten Fall zu einer globalen Erwärmung von $2,8^{\circ} \mathrm{C}$ gegen Ende des Jahrhunderts kommen wird. ${ }^{50}$ Der IPCC Sonderbericht $1,5^{\circ} \mathrm{C}$ globale Erwärmung macht deutlich, wie groß der Unterschied zwischen $1,5^{\circ} \mathrm{C}$ und $2^{\circ} \mathrm{C}$ Erderwärmung voraussichtlich sein wird und lässt daher erahnen, wie eine Welt bei $2,8^{\circ} \mathrm{C}$ Erwärmung aussehen könnte. Nicht zuletzt veranschaulicht aktuell die Corona-Krise, wie grundlegend der Ausstoß von $\mathrm{CO}_{2}$-Emissionen reduziert werden muss, damit wir die $1,5^{\circ} \mathrm{C}$-Ziel noch erreichen: Für 2020 wird aufgrund der Corona-Krise ein Rückgang des globalen Ausstoßes von Treibhausgasen um $8 \%$ prognostiziert, etwas mehr als alljährlich nötig wäre, um das $1,5^{\circ} \mathrm{C}-Z i e l$ noch zu erreichen. ${ }^{51}$

Diese Erkenntnisse wirken erdrückend, lassen aber nicht zwangsläufig den Schluss zu, dass die Entwicklung und der großskalige Einsatz von NETs unumgänglich sein werden. Aus dem Sein folgt grundsätzlich kein Sollen. NETs setzen vergleichsweise spät in der Wirkungskette des anthropogen verursachten Klimawandels an. Sie lassen sich daher mit einer Medizin vergleichen, die gegen die Folgen einer Erkrankung eingenommen wird, ihre Ursachen aber nicht beseitigt. Allerdings folgt aus den Fakten und Normen ebenso wenig, dass möglichen Potentiale der NETs für den Klimaschutz unbeachtet bleiben sollten. Insbesondere dort, wo NETs nachweislich umweltverträglich eingesetzt werden können, spricht a priori nichts gegen ihren Einsatz. Auch steht nicht fest, dass NETs in ihrer Umweltbilanz immer schlechter abschneiden als Maßnahmen, die auf die Vermeidung der Entstehung von Treibhausgasen abzielen. Strategisch gilt es aber im Blick zu behalten, dass das Klimaschutzrecht Maßnahmen, die auf die Vermeidung der Entstehung von Treibhausgasen gerichtet sind, gegenüber solchen der Rückhaltung und der Entnahme priorisiert. Damit trägt das Recht ihrem möglichen Beitrag zu Erreichung des wichtigsten Klimaschutzziels Rechnung, der Stabilisierung der Treibhausgaskonzentration in der Atmosphäre.

Open Access. Dieser Artikel wird unter der Creative Commons Namensnennung 4.0 International Lizenz veröffentlicht, welche die Nutzung, Vervielfältigung, Bearbeitung, Verbreitung und Wiedergabe in jeglichem Medium und Format erlaubt, sofern Sie den/die ursprünglichen Autor(en) und die Quelle ordnungsgemäß nennen, einen Link zur Creative Commons Lizenz beifügen und angeben, ob Änderungen vorgenommen wurden.

Die in diesem Artikel enthaltenen Bilder und sonstiges Drittmaterial unterliegen ebenfalls der genannten Creative Commons Lizenz, sofern sich aus der Abbildungslegende nichts anderes ergibt. Sofern das betreffende Material nicht unter der genannten Creative Commons Lizenz steht und die betreffende Handlung nicht nach gesetzlichen Vorschriften erlaubt ist, ist für die oben aufgeführten Weiterverwendungen des Materials die Einwilligung des jeweiligen Rechteinhabers einzuholen

Weitere Details zur Lizenz entnehmen Sie bitte der Lizenzinformation auf http://creativecommons.org/licenses/by/4.0/deed.de.

Open Access funding enabled and organized by Projekt DEAL.

48) Siehe z.B. Honneger/Reiner, The political economy of negative emissions technologies: consequences for international policy design, Clim. Policy 2018, 306; Bednar/Obersteiner/Wagner, On the financial viability of negative emissions, Nat. Commun. 2019, 1783.

49) Zur unionspolitischen Dimension siehe z. B. Geden/Schenuit, Unkonventioneller Klimaschutz - Gezielte $\mathrm{CO}_{2}$-Entnahme aus der Atmosphäre als neuer Ansatz der EU-Klimapolitik, 2020; siehe auch kurz European Academies Science Advisory Council, Negative emission technologies: What role in meeting the Paris Agreement targets?, 2018, S. $14 \mathrm{f}$.

50) Siehe Climate Action Tracker, Warming Projections Global Update December, 2019, S. 1.

51) Fulterer, Die Corona-Krise spart mehr $\mathrm{CO}_{2}$ als je zuvor, der Klimaschutz profitiert trotzdem nicht, Neue Zürcher Zeitung v. 5.5.2020. 